\title{
Downwash distribution of single-rotor unmanned agricultural helicopter on hovering state
}

\author{
Zhang Songchao, Xue Xinyu*, Sun Zhu, Zhou Lixin, Jin Yongkui \\ (Nanjing Research Institutes for Agricultural Mechanization, Ministry of Agriculture, Nanjing 210014, China)
}

\begin{abstract}
The effective coverage and velocity of downwash are directly related to the assemblage of spraying system and spraying effect. The downwash of the unmanned agricultural helicopter (UAH) N-3 was discussed in the paper. The computational fluid dynamics (CFD) methods were used to simulate and analyze the distribution of the downwash, and a wind field measurement device had been designed to test the downwash of UAH N-3. In the tests, the UAH N-3 was raised up to $5.0 \mathrm{~m}, 6.0 \mathrm{~m}$ and $7.0 \mathrm{~m}$ from the ground, "annular-radial-distribution- point" method was introduced, 8 directions separated by an angle of $45^{\circ}$ (the radial direction) with the intersection point of the main rotor shaft and the ground plane as the center, $0.5 \mathrm{~m}$ as the step length for the longitudinal (to $2.5 \mathrm{~m}$ ) and radial (to $4.0 \mathrm{~m}$ ) direction to set the sample points, considering the range of the rotor rotating circular area mainly. The $5 \mathrm{~m}$ height results of N-3 were fully discussed to describe the downwash distribution with the longitudinal altitude increased and the radial distance increased. The standard deviations of five test altitudes for eight directions were comparatively analyzed, the results showed that the total standard deviation was not greater than $0.6 \mathrm{~m} / \mathrm{s}$. The overall relative maximum margin of error calculated from the simulation and measurement data was between 0.6 and 0.7 , which verified the credibility of the simulation data. High-order polynomials were used to fitting the simulation and measurement data, the fitting results showed that the polynomial coefficient of determination $R^{2}$ met or exceeded 0.75 when the altitudes were more than $1 \mathrm{~m}$, indicating the fit equation having the reference values. When the altitudes equal or less than $0.5 \mathrm{~m}$, the polynomial coefficient of determination $R^{2}$ was smaller, ranging during 0.3 to 0.7 . The study would provide some foundations for the optimization of the assemblage of spraying system on the single-rotor UAH, which would promote China aviation plant protection.
\end{abstract}

Keywords: unmanned agricultural helicopter, single rotor, CFD simulation, downwash distribution, spraying effect DOI: $10.25165 /$ j.ijabe.20171005.3079

Citation: Zhang S C, Xue X Y, Sun Z, Zhou L X, Jin Y K. Downwash distribution of single-rotor unmanned agricultural helicopter on hovering state. Int J Agric \& Biol Eng, 2017; 10(5): 14-24.

\section{Introduction}

UAH can efficiently accomplish the spraying application above the crops without restrictions generally

\section{Received Date: 2017-04-18 Accepted date: 2017-09-15}

Biographies: Zhang Songchao, MS, research interests: precise pesticide spraying, Email: 51832898@qq.com; Sun Zhu, BS, research interests: detection and control, Email: 43148588@qq.com; Zhou Lixin, ME, research interests: crop protection machinery, Email: 525751791@qq.com; Jin Yongkui, ME, research interests: mechanical design and theory, Email: 120059323@qq.com.

* Corresponding author: Xue Xinyu, PhD, Professor, research interest: crop protection and machinery engineering. Mailing address: Nanjing Research Institute for Agricultural Mechanization, Ministry of Agriculture, Nanjing 210014, China. Tel: +86-2584346243, Email: 735178312@qq.com. of the crop species or the growth period because of low requirements for airspace ${ }^{[1,2]}$. The high speed rotating rotors push the air downward to ensure the takeoff and flight for the UAH. Meanwhile, the downwash generated by the rotors could assist in the droplets deposition on the leaves and penetration into the canopy. The coverage and velocity of the downwash will directly influence the effect of the aerial spraying application ${ }^{[3,4]}$. Qin et al. ${ }^{[5,6]}$ developed the unmanned aerial vehicle and carried out the aerial spraying experiments on corns with the N-3. In the experiments a certain concentration solution of Rhodamine-B was used as an alternative for pesticides. The flight altitudes were changed during the process of spraying while other conditions remained constant, and the conclusions obtained was that when the 
flight altitude was $7.0 \mathrm{~m}$, the total amount of droplet deposition was more than that of $5.0 \mathrm{~m}$ and $9.0 \mathrm{~m}$ with the downwash assistant. Xue et al. ${ }^{[7]}$ tested the control effects of rice planthoppers and leafrollers with UAH aerial spraying application. The results showed that the control effects after spraying $3 \mathrm{~d}, 5 \mathrm{~d}, 10 \mathrm{~d}$, were all better than the effects of traditional stretcher sprayer. Zhang et al. ${ }^{[8]}$ studied the droplet deposition levels internal the canopy with the downwash assistant of aerial spraying, and proposed a new computer simulation method to calculate the droplet drift amount with the verification experiments. It was concluded that the upper canopy layer (rice) deposited amount of droplets was about $28 \%$ of the total spray volume, and the under canopy layer was about $26 \%$, the drift amount was $12.9 \%$ which $90 \%$ occurred with $8 \mathrm{~m}$ from the target area.

Research related to unmanned agricultural helicopter wind field is limited. Domestic and foreign scholars have developed momentum theory ${ }^{[9,10]}$, blade element theory ${ }^{[11]}$, rotor vortex theory ${ }^{[12]}$, etc., to guide the helicopter rotor design improving the control flexibility and lift force ${ }^{[13]}$. Zhao et al. ${ }^{[14]}$ had established a 3-D Euler equations model to simulate the rotor flow for missile emission. Ren et al. ${ }^{[15]}$ studied the impacts the rotor downwash to the exhaust gas using the Fluent software based the blade element theory. Although these studies focused on the helicopter rotor aerodynamic characteristics, there are some certain reference values for UAH downwash researches. Manned fixed-wing agricultural aircrafts have been widely used in some developed countries ${ }^{[16]}$, while research related to the $\mathrm{UAH}$ is rare until recent years. Lee et al. ${ }^{[17,18]}$ set color mist smoke as the display carriers to observe the wind field motion pattern. Thomson et al. ${ }^{[19]}$ found the spray deposition and drift differences under the rotor wind field between the right and the left of the fuselage. Li et al. $^{[20-22]}$ developed a wireless test system to measure the UAH wind field for optimizing the parameters in terms of hybrid rice pollination. Zhang et al. ${ }^{[23]}$ studied the near ground wake of fixed-wing aircraft Trush 510G to optimize the aerial spraying.

Currently, there are research results on the helicopter wind field domestic and overseas, there are very few researches on analyzing the downwash of UAH, especially on the downwash coverage, velocity and relationship with the assembly of spraying equipment, flight altitude, effective spay width, etc.

The computational fluid dynamics (CFD) methods have been used in the spraying field ${ }^{[24,25]}$. This research uses computational fluid dynamics (CFD) methods to simulate the rotor wind field of UAH N-3, in which the downwash is focused on. A wind field measurement device has been designed to test the downwash of UAH N-3. The downwash close to the ground which as a main factor to the droplet deposition has been systematically measured and analyzed, in order to ascertain the downwash distribution for optimizing the assembly of spraying equipment and the aerial spraying parameters.

\section{Materials and methods}

\subsection{Model establishment}

The UAH N-3 shows as Figure 1, it is a single-rotor, oil engine unmanned helicopter, the detailed parameters shown in Table 1, the main physical dimension measured shows in Figure 2, aerial spraying parameters refer to references 8 .

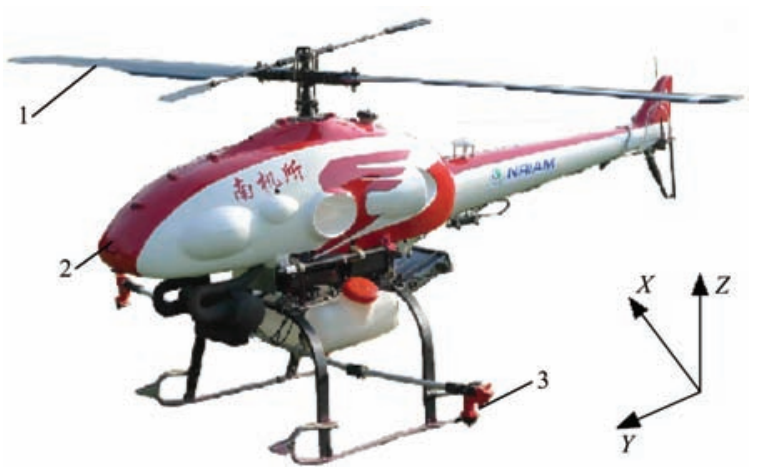

1. Rotor 2. Fuselage 3. Centrifugal atomization nozzles

Figure 1 Unmanned agricultural helicopter, N-3

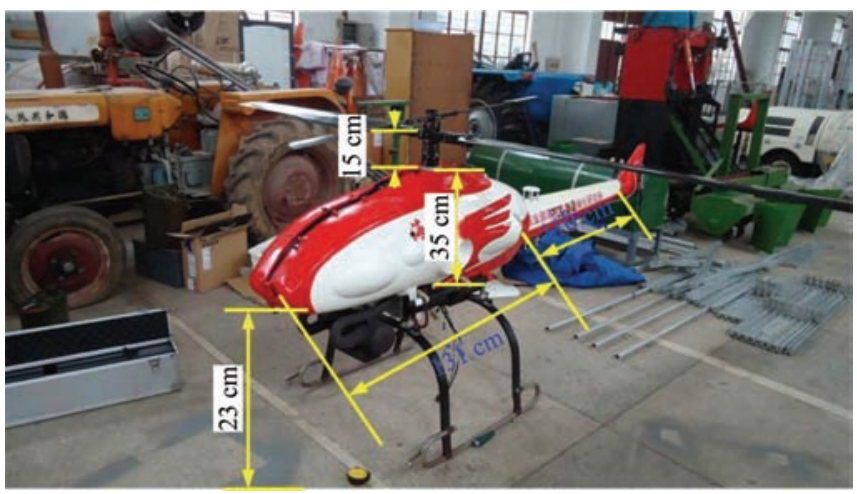

Figure 2 Main physical dimension 
Table 1 Main parameters of N-3

\begin{tabular}{lcc}
\hline \multicolumn{1}{c}{ Parameters } & Values & Remarks \\
\hline Main rotor diameter/mm & 3115 & Carbon fiber \\
Size/mm & $2696 / 720 / 1109$ & Length $/$ width $/$ Height \\
Takeoff weight $/ \mathrm{kg}$ & 100 & Max weight \\
Blade installation angle $/\left(^{\circ}\right)$ & $2.4,12.1$ & Min and max values \\
Blade twist angle $/\left(^{\circ}\right)$ & -6 & \\
Engine speed $/ \mathrm{r} \cdot \mathrm{min}^{-1}$ & 6350 & Rated speed \\
Engine power $/ \mathrm{HP}$ & 25 & Max power \\
Rotor speed $/ \mathrm{r} \cdot \mathrm{min}^{-1}$ & 828 & Rated speed \\
\hline
\end{tabular}

The CFD modeling methods and establishing process mainly includes meshing the body (fuselage) in the pre-processing software Gambit and surface transition with non-uniform B-spline (NURBS) with more details in reference 8 . The body meshes show in Figure 3.

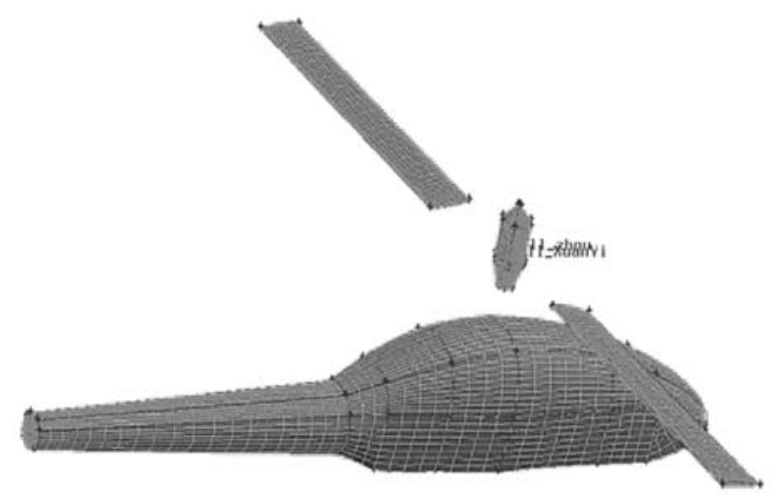

Figure 3 Meshes of N-3

\subsection{Downwash simulation}

The following equation is the general form of Navier-Stokes equation:

$$
\begin{aligned}
& \frac{\partial(\rho \psi)}{\partial t}+\frac{\partial(\rho u \psi)}{\partial x}+\frac{\partial(\rho v \psi)}{\partial y}+\frac{\partial(\rho w \psi)}{\partial z}= \\
& \frac{\partial}{\partial x}\left(\zeta \frac{\partial \psi}{\partial x}\right)+\frac{\partial}{\partial y}\left(\zeta \frac{\partial \psi}{\partial y}\right)+\frac{\partial}{\partial z}\left(\zeta \frac{\partial \psi}{\partial z}\right)+S
\end{aligned}
$$

where, $\psi$ means a universal variable; $\zeta$ means generalized diffusion coefficient; $S$ means a generalized source term.

In the standard $k-\omega$ model, there are no requirements for additional damping term near the boundary wall, and the normal distance defined to the boundary wall, suitable for complex boundaries. The sst model is more sensitive to the strength of the vortex flow. In this research, $k \omega$-sst turbulence model was introduced. The $k \omega$-sst turbulence model was developed from the standard $k$ - $\omega$ model with adjustments of the turbulent viscosity definition according to the shear-stress transport, and the adjustments of appropriate constants, in additional adding a damping dissipative term in $\omega$ equation. Definitions and expressions of $\mathrm{k} \omega$-sst turbulence model show as below.

According to dimensionless form, $\rho \kappa$ and $\rho \omega$ conservative transport equations can be expressed as:

$$
\begin{aligned}
& \frac{\partial \rho k}{\partial t}+\frac{\partial \rho k v_{j}}{\partial x_{j}}-\frac{\partial}{\partial x_{j}}\left(\frac{\Gamma_{k}}{\operatorname{Re}_{r}} \frac{\partial k}{\partial x_{j}}\right)=\frac{\mu_{t}}{\operatorname{Re}_{r}} S^{2}-\rho \beta^{*} k \omega \\
& \frac{\partial \rho \omega}{\partial t}+\frac{\partial \rho \omega v_{j}}{\partial x_{j}}-\frac{\partial}{\partial x_{j}}\left(\frac{\Gamma_{\omega}}{\operatorname{Re}_{r}} \frac{\partial \omega}{\partial x_{j}}\right)=\rho \alpha S^{2}-\rho \beta \omega \omega \\
& +2\left(1-F_{1}\right) \rho \sigma_{\omega, 2} \frac{1}{\omega} \frac{\partial k}{\partial x_{j}} \frac{\partial \omega}{\partial x_{j}}
\end{aligned}
$$

The $k \omega$-sst turbulence equation variable coefficients are defined and expressed as:

$$
\begin{aligned}
& \Gamma_{k}=\mu+\frac{\mu_{t}}{\sigma_{k}} \\
& \Gamma_{\omega}=\mu+\frac{\mu_{t}}{\sigma_{\omega}}
\end{aligned}
$$

where,

$$
\begin{gathered}
\sigma_{k}=\frac{1}{F_{1} / \sigma_{k, 1}+\left(1.0-F_{1}\right) / \sigma_{k, 2}} \\
\sigma_{\omega}=\frac{1}{F_{1} / \sigma_{\omega, 1}+\left(1.0-F_{1}\right) / \sigma_{\omega, 2}}
\end{gathered}
$$

The $k \omega$-sst turbulence equation dynamic viscous coefficient coefficients are defined and expressed as:

$$
\begin{aligned}
& \frac{\mu_{t}}{\mathrm{Re}_{r}}=\frac{\rho k}{\omega} \frac{1}{\max \left[\frac{1}{\alpha^{*}}, \frac{\Omega F_{2}}{a_{1} \omega}\right]} \\
& \alpha^{*}=\alpha_{\infty}^{*}\left(\frac{\alpha_{0}^{*}+\mathrm{Re}_{t} / R_{k}}{1+\mathrm{Re}_{t} / R_{k}}\right)
\end{aligned}
$$

where,

$$
\operatorname{Re}_{t}=\operatorname{Re}_{r} \frac{\rho k}{\mu \omega}, \quad \alpha_{0}^{*}=\frac{\beta_{i}}{3}
$$

Production item of equation k:

$$
\text { production }_{k}=\mu_{t} S^{2} \frac{1}{\operatorname{Re}_{r}}
$$

Production item of equation $\omega$ :

$$
\text { production }_{\omega}=\rho \alpha S^{2} \frac{1}{\mathrm{Re}_{r}}
$$

where,

$$
\alpha=\frac{\alpha_{\infty}}{\alpha^{*}}\left(\frac{\alpha_{0}+\mathrm{Re}_{t} / R_{k}}{1+\mathrm{Re}_{t} / R_{k}}\right)
$$

Mixing functions $F_{1}$ and $F_{2}$ were introduced to revise the standard model, while calculating far away from the 
boundary $F_{1} \rightarrow 0, F_{2} \rightarrow 0$, near the boundary $F_{1} \rightarrow 1, F_{2} \rightarrow 1$.

The main values of $k \omega$-sst turbulence model are shown in Table 2.

Table 2 Main parameters of N-3

\begin{tabular}{cccccc}
\hline Parameters & Values & Parameters & Values & Parameters & Values \\
\hline$\alpha_{\infty}^{*}$ & 1 & $R_{\beta}$ & 8 & $a_{1}$ & 0.31 \\
$\alpha_{\infty}$ & $F_{1} \alpha_{\infty, 1}+\left(1.0-F_{1}\right) \alpha_{\infty, 2}$ & $R_{k}$ & 6 & $\beta_{i}$ & 0.072 \\
$\alpha_{\infty, 2}$ & $\frac{\beta_{i, 1}}{\beta_{\infty}^{*}}-\frac{k^{2}}{\sigma_{w, 1} \sqrt{\beta_{\infty}^{*}}}$ & $\alpha_{0}$ & 0.111 & $\beta_{i, 1}$ & 0.075 \\
& $\frac{\beta_{i, 1}}{\beta_{\infty}^{*}}-\frac{k^{2}}{\sigma_{w, 1} \sqrt{\beta_{\infty}^{*}}}$ & $R_{\omega}$ & 2.95 & $\beta_{i, 2}$ & 0.0828 \\
$\alpha_{\infty, 1}$ & 0.09 & $\sigma_{k, 1}$ & 1.176 & $M_{t 0}$ & 0.25 \\
$\beta_{\infty}^{*}$ & 1.5 & $\sigma_{\omega, 1}$ & 2 & $\sigma_{\omega, 2}$ & 1.168 \\
$\xi^{*}$ & & 1 & & & \\
$\sigma_{k, 2}$ & & & & & \\
\hline
\end{tabular}

The initial conditions set as the atmospheric temperature $303 \mathrm{~K}$, far-field static atmospheric pressure $101.325 \mathrm{kPa}$, assuming the air was not compressed during the calculations.

\subsection{Downwash measurements}

\subsubsection{Measurement device}

The measurement device chose the Kestrel 4500 anemometer produced by the Nielsen-Kellerman company, US. The maximum range could be $40 \mathrm{~m} / \mathrm{s}$ with resolution of $0.1 \mathrm{~m} / \mathrm{s}$, the measurement accuracy is $\pm 3 \%$ power by 2 AAA batteries. About 2900 data could be measured and stored in the internal memory. The data collected could be read out to PC by the Kestrel Interface for convenient analysis.

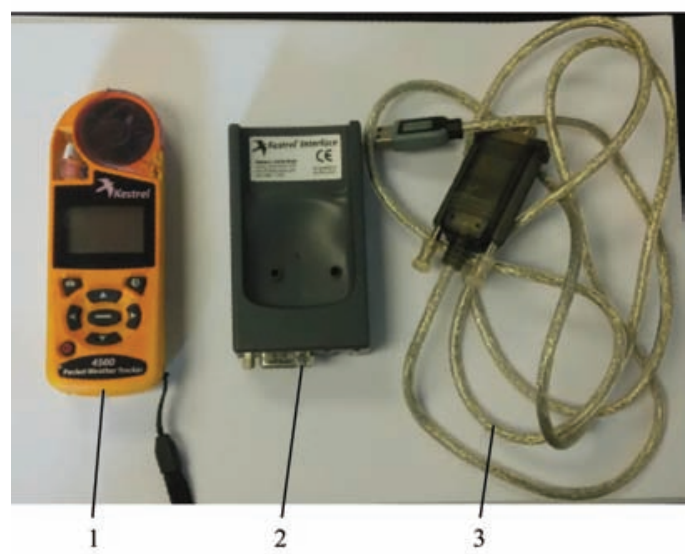

$\begin{array}{lll}\text { 1. Host of Kestrel } 4500 & \text { 2. Data reading terminal-Kestrel Interface 3. Data cable }\end{array}$

Figure 4 Anemometer and related components

\subsubsection{Experimental device design}

The experimental device is mainly made up of a single column mobile hydraulic lifting mechanism with four universal wheels, the direction traction wheel and the handle installed on the bottom, which the lifting range is $1-10 \mathrm{~m}$, the maximum load is $200 \mathrm{~kg}$ powered by a $2.5 \mathrm{~kW}$ AC motor. On the top of the hydraulic telescopic cylinder, a $0.8 \times 0.8 \mathrm{~m}^{2}$ stainless steel mounting platform is installed with two parallel grooved aluminum strips aside to fix the UAH landing gear on the $0.5 \mathrm{~m} \times$ $0.06 \mathrm{~m} \times 0.06 \mathrm{~m}$ aluminum materials by T-bolts. Four rigid cables connect with the platform to the ground for adjusting the stability of the hydraulic lifting mechanism. Meanwhile, in order to ensure the safety and convenience of the experiments, a concrete geosyncline $(12.0 \mathrm{~m} \times$ $2.0 \mathrm{~m} \times 1.5 \mathrm{~m}$ ) was built to place the experimental device and get the platform down to the ground before experiments. The experimental device and installation are shown in Figure 5.

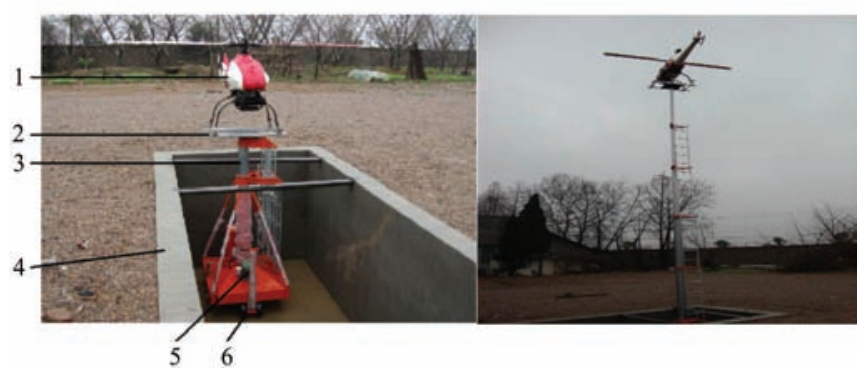

1. UAH 2. Work platform 3. Hydraulic telescopic column 4. Cement geosynclines 5. AC motor 6. Direction traction and handle

Figure 5 Experimental device and installation

\subsubsection{Test site}

The test site locates in eastern laboratory, Nanjing Research Institute of Agricultural Mechanization $\left(118^{\circ} 52^{\prime} 20.58^{\prime \prime} \mathrm{E}, \quad 30^{\circ} 02^{\prime} 39.38^{\prime \prime} \mathrm{W}\right)$. The average temperature was $30.2^{\circ} \mathrm{C}$, the average relative humidity was $71.2 \%$, the wind speed was less than $0.5 \mathrm{~m} / \mathrm{s}$.

\subsubsection{Test direction definition}

The downwash tested was guidable to aerial spraying. Considering the crops as rice, wheat, corn et al. are usually not more than or about $2.0 \mathrm{~m}$, and the UAH N-3 flight height is usually not more than $7.0 \mathrm{~m}$, so in the experiments, "annular-radial-distribution-point" method was introduced, the UAH N-3 was raised up to $5.0 \mathrm{~m}$, $6.0 \mathrm{~m}$ and $7.0 \mathrm{~m}$ from the ground, "annular-radialdistribution-point" method was introduced, 8 directions separated by an angle of $45^{\circ}$ (the radial direction) with the intersection point of the main rotor shaft and the ground plane as the center, $0.5 \mathrm{~m}$ as the step length for the longitudinal (to $2.5 \mathrm{~m}$ ) and radial (to $4.0 \mathrm{~m}$ ) direction 
to set the sample points. Figure 1 shows the three-dimensional coordinate system, which the intersection of the rotor shaft and the ground plane is as the circle center $O$, the $X$-axis positive axle direction as the a starting direction D1, at interval of $45^{\circ}$ counterclockwise looking down setting a measurement direction, following as D2, D3, D4, D5, D6, D7 and D8, shows as Figure 6.
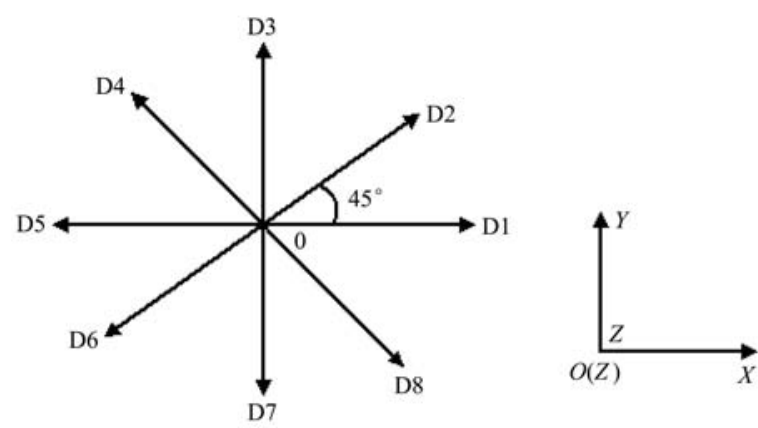

Figure 6 Definition of measuring directions

\subsubsection{Sampling points arrangement and data collect}

The sampling points were where the downwash velocity collected. During the experiments, the Kestrel 4500 was fixed on an aluminum bar $(3.0 \mathrm{~m} \times 0.06 \mathrm{~m} \times$ $0.06 \mathrm{~m})$ with an interval of $0.5 \mathrm{~m}(\mathrm{t} 1, \mathrm{t} 2, \mathrm{t} 3, \mathrm{t} 4, \mathrm{t} 5)$, and the bar was moved as a step length (a, b, c, d, e, f, g, h on D1-D8). Figure 7 shows the left side view for the sampling points arrangement (direction from the negative $X$-axis to the positive).

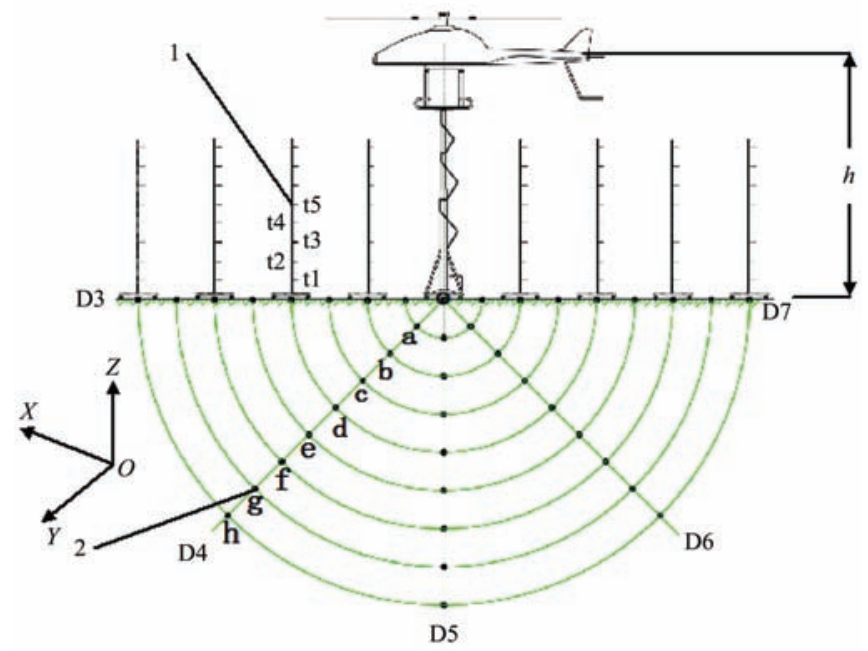

1. Schematic of longitudinal measurement $\quad 2$ Schematic of radial measurement Figure 7 Definition of measuring directions

The Kestrel 4500 was placed horizontally, mode was set as automatic storage. The test began when the N-3 was working under the rated condition (Table 1) raised up to $5.0 \mathrm{~m}, 6.0 \mathrm{~m}, 7.0 \mathrm{~m}$ respectively. Every one minute three times were repeated on each sampling point, the average velocity value was chosen. The concrete geosynclines were sealed with wood slabs to reduce the impact on the collected data.

\section{Results and analysis}

\subsection{Analysis of the simulation data}

The Figure 8 shows the wind field vector distribution diagram of N-3 at height $5.0 \mathrm{~m}$. The downwash velocity was focused on.

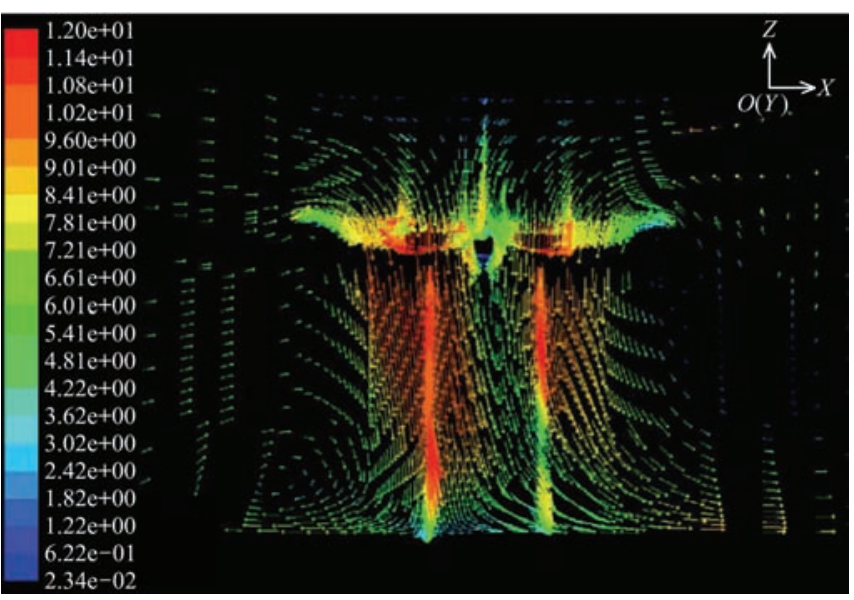

Figure 8 Vector diagram of simulated wind field, $5 \mathrm{~m}$

According to the simulation results, the downwash played a main role in the wind field distribution on hovering state. At the range of the rotor, there is a vortex phenomenon as expected. The wind velocity values were among 0.622-12.08 $\mathrm{m}$, during which the downwash velocities bigger distributed among 1/4-3/4 length of the covered range by the rotor. The downwash velocity values appeared a trend of becoming big then small radially because of the fuselage block. The velocity near the ground $(<0.5 \mathrm{~m})$ varied not obviously because of "ground effect". Provided that some assumptions were made in the CFD simulations, so the simulation results were not significant and the variations were consistent when the N-3 values were set at $5.0 \mathrm{~m}$, $6.0 \mathrm{~m}$, and $7.0 \mathrm{~m}$ in height.

\subsection{Analysis of the experimental data}

\subsubsection{Standard deviation analysis}

According to the methods above, the N-3 values were raised up to $5.0 \mathrm{~m}, 6.0 \mathrm{~m}$ and $7.0 \mathrm{~m}$ to measure the downwash velocities. The standard deviations of the data were calculated according to the equation below, the results are shown in Figure 9. 


$$
\sigma=\sqrt{\frac{1}{N} \sum_{i=1}^{N}\left(x_{i}-u\right)^{2}}
$$

where, $\sigma$ is the standard deviation, $\mathrm{m} / \mathrm{s}$ as the unit; $N$ is the number of the samples; $x_{i}$ is the value of a sample, $\mathrm{m} / \mathrm{s}$ as the unit; $u$ is the average value of sample.
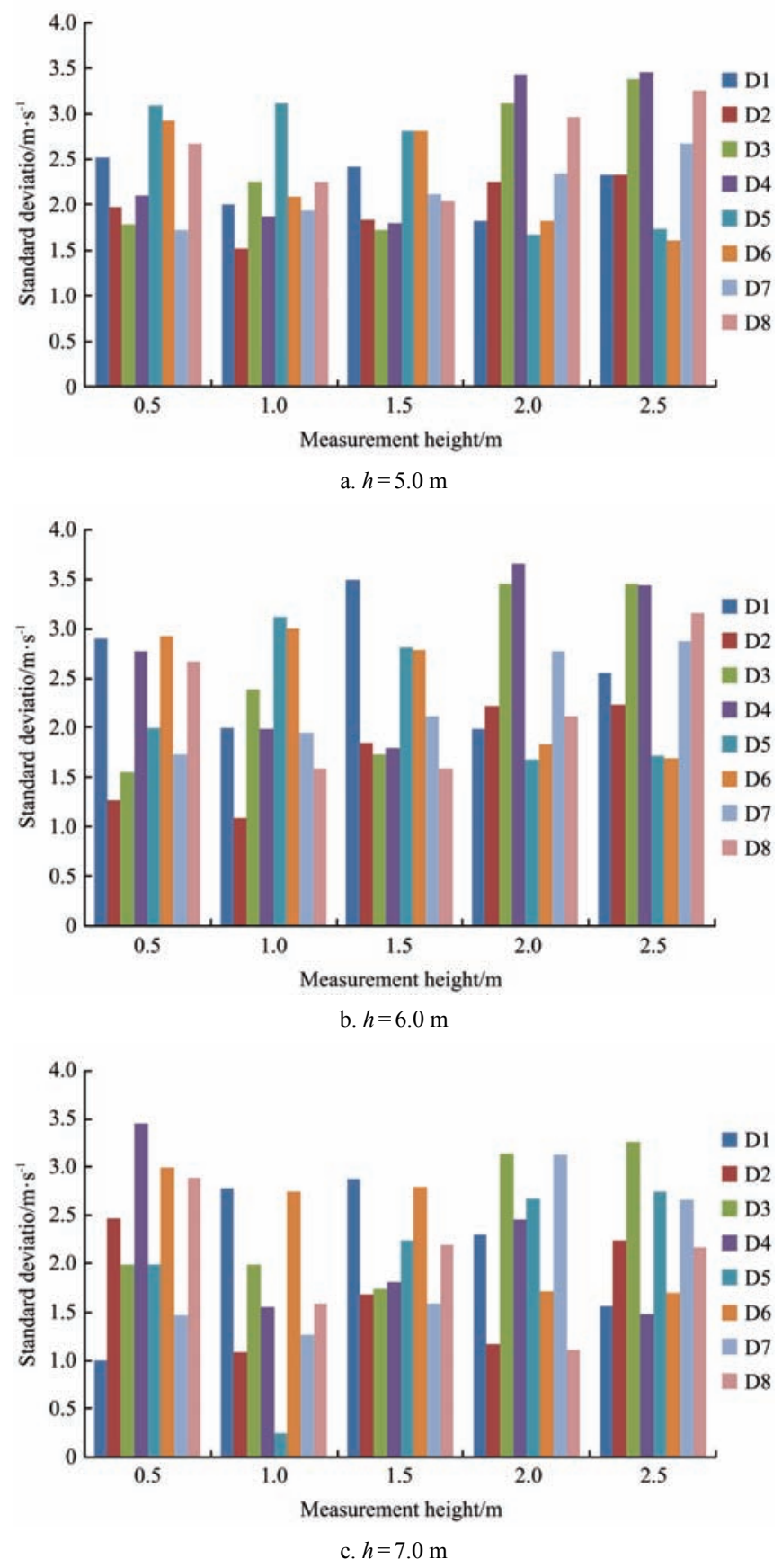

Figure 9 Sample standard deviation of different heights

As showed that most of the standard deviations were less than $2 \mathrm{~m} / \mathrm{s}$, very few deviations were greater than $3.5 \mathrm{~m} / \mathrm{s}$.

Comparison of the overall deviations under $5 \mathrm{~m}, 6 \mathrm{~m}$ and $7 \mathrm{~m}$ in heights, the results showed that the overall deviations of three heights were all less than $1 \mathrm{~m} / \mathrm{s}$ which meant the data obtained could characterize the downwash velocity distribution conditions accurately. The $\sigma_{0.5 \mathrm{~m}}<$ $\sigma_{6.5 \mathrm{~m}}<\sigma_{7.5 \mathrm{~m}}$ showed (Figure 10) that the dispersion degree of the data obtained when the UAH was $5 \mathrm{~m}$ were more smaller which meant more accurately to characterize the downwash velocity distribution. So the data obtained under $5 \mathrm{~m}$ were analyzed in the paper.

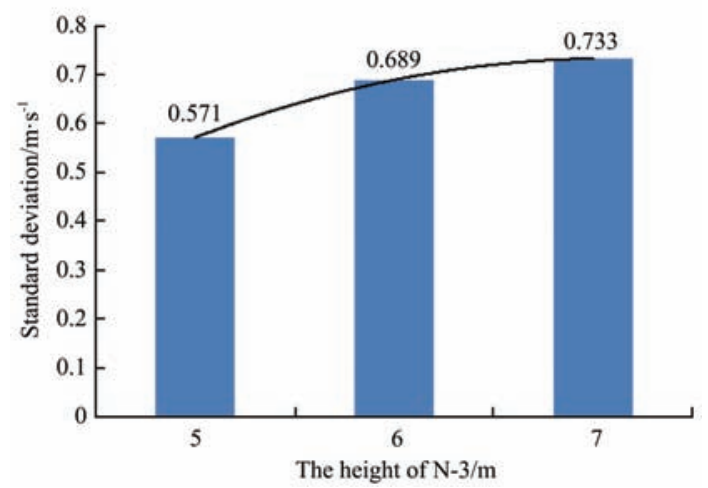

Figure 10 Overall sample standard deviation comparison of $5.0 \mathrm{~m}, 6.0 \mathrm{~m}, 7.0 \mathrm{~m}$

\subsubsection{Downwash analysis along radial distance}

Figures 11 show the data statistic trend of the D1 to D8 when the UAH was raised up to $5 \mathrm{~m}$ with $0.5 \mathrm{~m}$, $1.0 \mathrm{~m}, 1.5 \mathrm{~m}, 2.0 \mathrm{~m}$ and $2.5 \mathrm{~m}$ measurement heights.

From the Figures, as can been seen that: (1) the downwash change trend was from small to bigger then smaller as the radial distance increased especially when the measurement heights were $2.0 \mathrm{~m}$ and $2.5 \mathrm{~m}$ the trend were more obvious, which kept a good consistency with the simulation trend; (2) The max value of the downwash was $11.37 \mathrm{~m} / \mathrm{s}$, the values of the downwash bigger relatively distributed among radial distance $0.5-1.5 \mathrm{~m}$, about a quarter to three quarters of the rotor radius length $(0.38-1.168 \mathrm{~m})$, consistent with the simulation results; (3) The impact of the "ground effect" to the measurement results mainly reflected in the trend curve of $0.5 \mathrm{~m}$ measurement height, which was most fluctuant with the weakest monotonicity compared others.

\subsubsection{Downwash analysis along vertical distance}

The Figure 12 shows the data statistic trend of different step length points ( $a, b, c, d, e, f, g, h)$ when the UAH was raised up to $5.0 \mathrm{~m}$ with $0.5 \mathrm{~m}, 1.0 \mathrm{~m}, 1.5 \mathrm{~m}$, $2.0 \mathrm{~m}$ and $2.5 \mathrm{~m}$ in measurement heights.

Results obtained by the vertical analysis showed that: (1) The downwash value increased as the measurement height increased (55 curves coincide with the trend in a total of 66) on the same point (such as D1-a); (2) The 
downwash values differed with the same radial and vertical distance on different step points, and the maximum could be about $10.0 \mathrm{~m} / \mathrm{s}$ (D5-d and D4-d, vertical distance $2 \mathrm{~m}$ ); (3) The curves appeared a concave shape at about $1 \mathrm{~m}$ height which indicated the
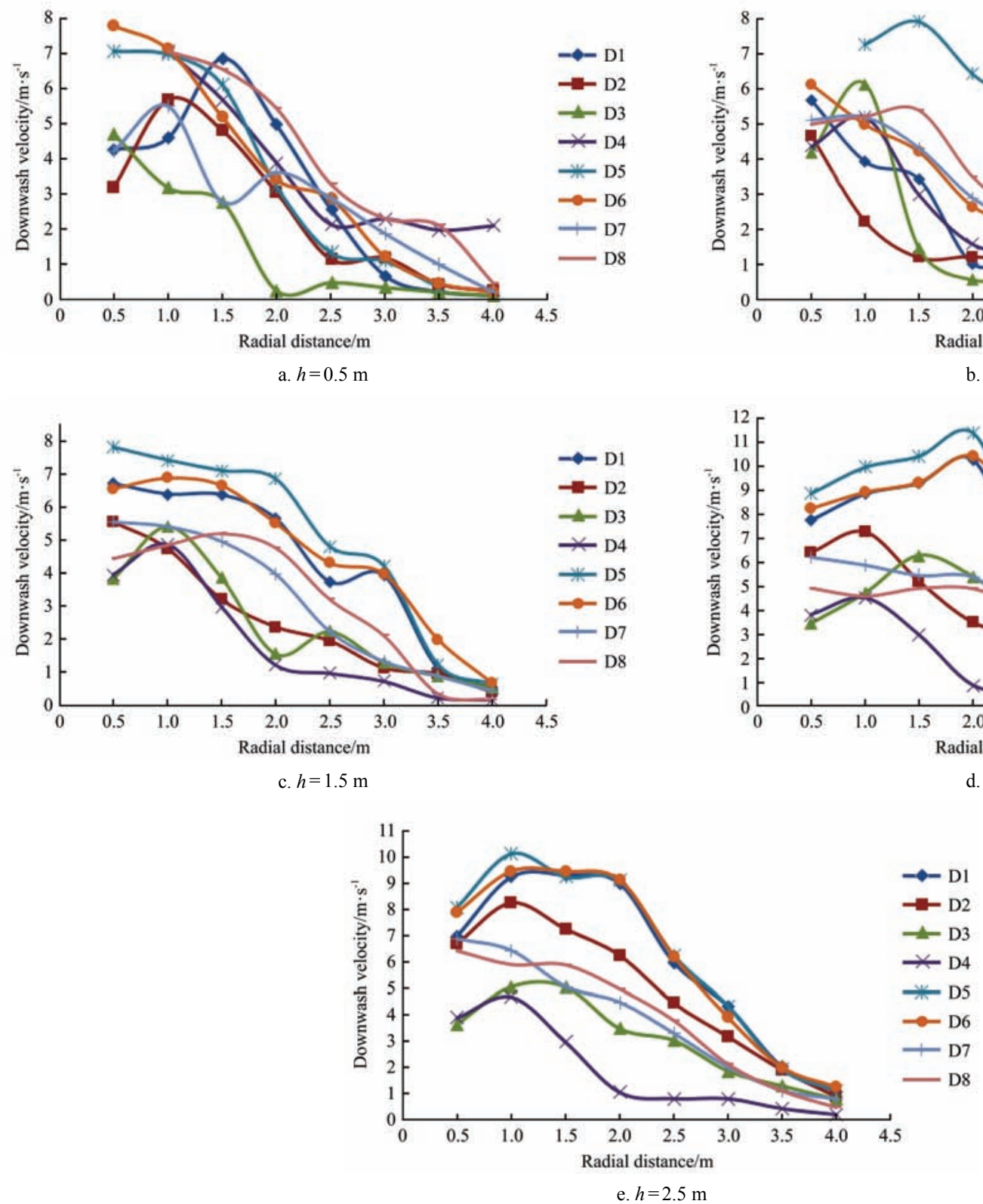

Figure 11 Downwash of different measurement height

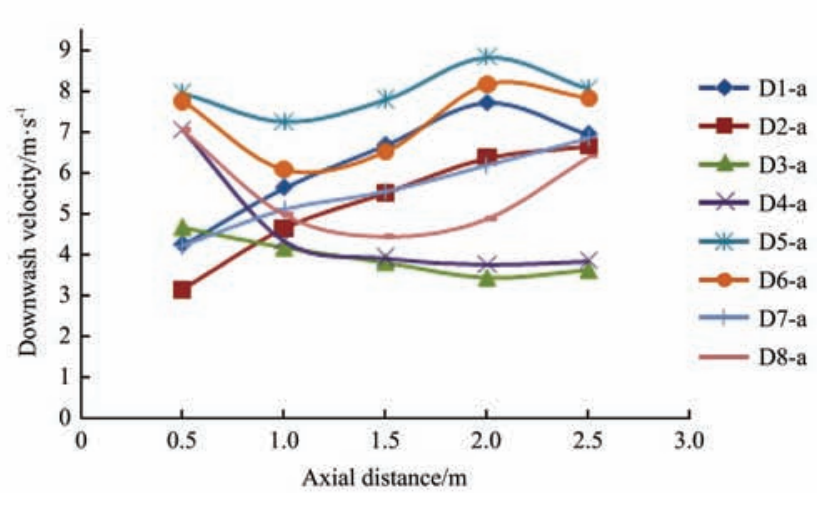

a. Downwash of point a downwash occurred a presence of local minimal interval about $1 \mathrm{~m}$ height; (4) The curves appeared a convex shape at about $2 \mathrm{~m}$ height which indicated the downwash occurred a presence of local maximum interval about $2 \mathrm{~m}$ in height.
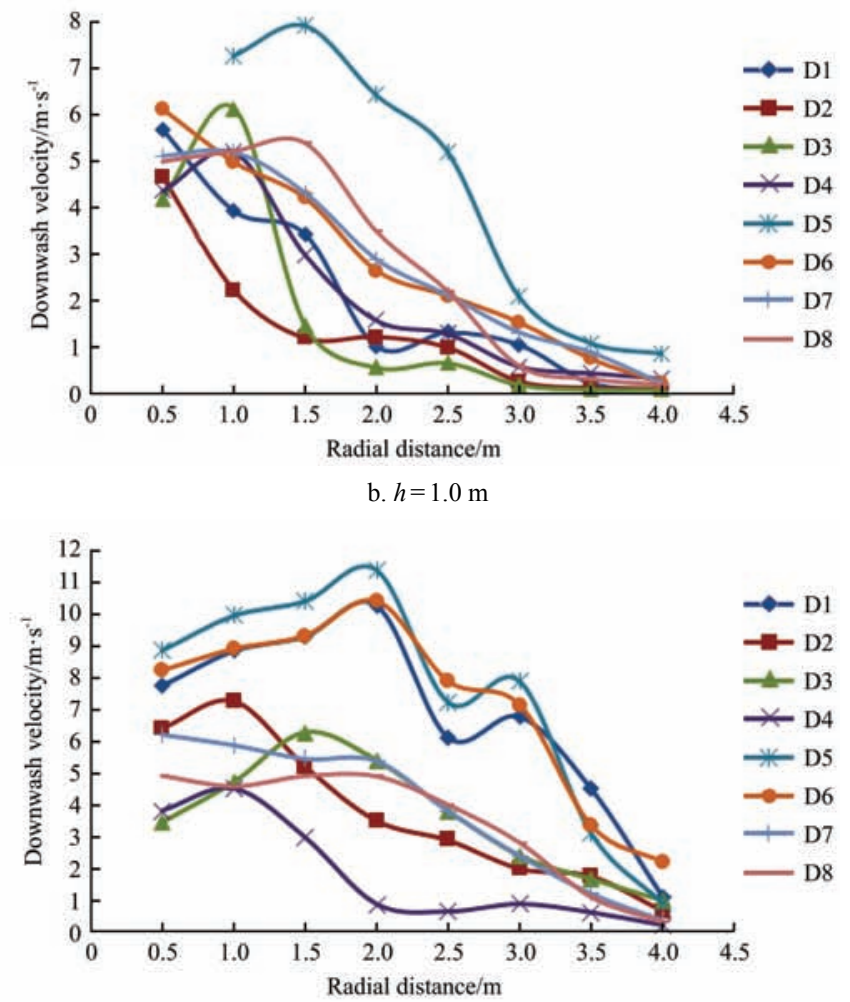

d. $h=2.0 \mathrm{~m}$

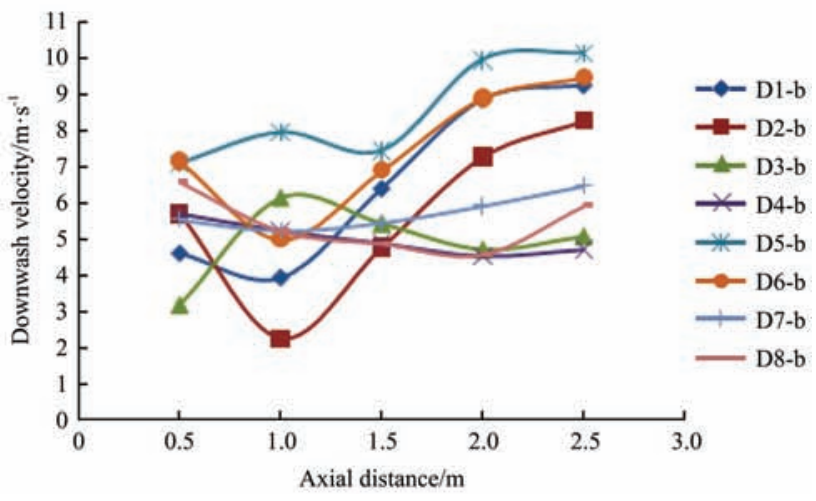

b. Downwash of point $b$ 

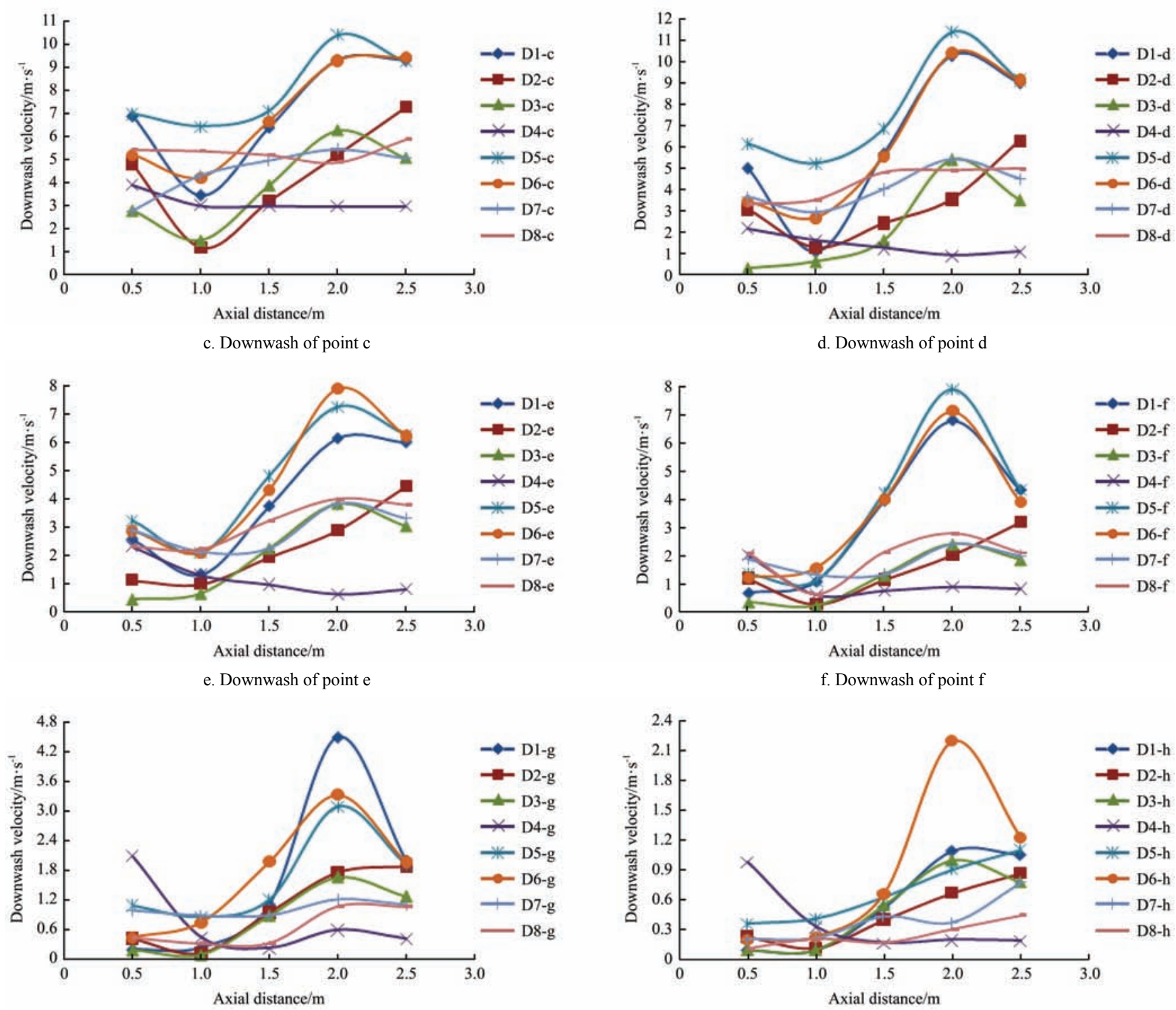

g. Downwash of point $g$

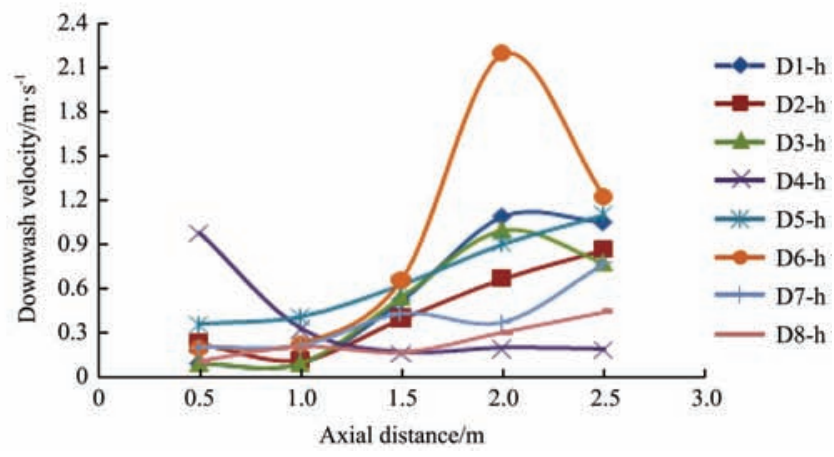

Figure 12 Downwash data statistic trend of different step length points

\subsection{Comparative analysis of the data}

\subsubsection{Error analysis}

The downwash distribution data were obtained by CFD simulation and experiments. Here took the experimental data as the exact value considering the assumptions in the simulation, the relative error margin of simulation and the experimental results was defined as below.

$$
e=\frac{\left|q_{e}-q_{s}\right|}{q_{e}}
$$

where, $q_{e}$ is the experimental value, $\mathrm{m} / \mathrm{s} ; q_{s}$ is the simulation value, $\mathrm{m} / \mathrm{s}$.

The Table 3 showed all the data and the relative error margin of D1.

From the data in the Table 3 the conclusion could be drawn that: (1) The simulation data values were close or bigger almost, maybe because some assumptions such as the air incompressibility, the ambient temperature, the ground airflow bounce had impacts to the downwash, which were ignored in the simulation while existed in the experiments, that was why the experimental values are more accurate. (2) The measurement values and the simulation values appeared alternately more obviously when the measurement height was $0.5 \mathrm{~m}$ which verified the presence of ground effect mentioned herein before. (3) Except the $0.5 \mathrm{~m}$ height, the relative error margins were not big, and the overall maximum one was less than 0.70 , which the smallest one was 0.030 . The analysis results within 3 radial distances were more apparent which verified the degree of confidence simulation data 
to experimental ones.

\subsubsection{Curve fitting}

Based on the data in Table 3, polynomial curve fitting analysis was carried out, the polynomials and the coefficients of determination $R^{2}$ were as follow in Figure 13.

Table 3 Comparison of simulated and experimental results (D1)

\begin{tabular}{|c|c|c|c|c|c|c|c|c|c|}
\hline \multirow{2}{*}{$\begin{array}{l}\text { Measurement } \\
\text { height } \mathrm{h} / \mathrm{m}\end{array}$} & \multirow{2}{*}{$\begin{array}{c}\text { Relative error margin } \\
\text { values } / \mathrm{m} \cdot \mathrm{s}^{-1}\end{array}$} & \multicolumn{8}{|c|}{ Radial distance/m } \\
\hline & & 0.5 & 1.0 & 1.5 & 2.0 & 2.5 & 3.0 & 3.5 & 4.0 \\
\hline \multirow{3}{*}{0.5} & $\mathrm{q}_{\mathrm{e}}$ & 4.27 & 4.60 & 6.87 & 4.29 & 2.57 & 0.67 & 0.21 & 0.10 \\
\hline & $\mathrm{q}_{\mathrm{s}}$ & 2.48 & 3.59 & 6.29 & 6.36 & 5.21 & 3.76 & 2.11 & 1.22 \\
\hline & $\mathrm{e}$ & 0.419 & 0.219 & 0.084 & 0.482 & 1.027 & 4.611 & 9.047 & 11.200 \\
\hline \multirow[b]{2}{*}{1.0} & $\mathrm{q}_{\mathrm{e}}$ & 5.67 & 3.93 & 3.43 & 1.03 & 1.33 & 1.07 & 0.25 & 0.10 \\
\hline & $\mathrm{q}_{\mathrm{s}}$ & 8.69 & 6.01 & 7.21 & 5.32 & 1.82 & 0.89 & 0.56 & 0.23 \\
\hline \multirow{3}{*}{1.5} & $\mathrm{q}_{\mathrm{e}}$ & 6.71 & 6.39 & 6.37 & 5.65 & 3.73 & 3.93 & 1.12 & 0.53 \\
\hline & $\mathrm{q}_{\mathrm{s}}$ & 6.01 & 8.29 & 4.65 & 4.81 & 4.22 & 3.46 & 2.11 & 1.76 \\
\hline & $\mathrm{e}$ & 0.104 & 0.123 & 0.142 & 0.148 & 0.131 & 0.119 & 0.883 & 2.321 \\
\hline \multirow{3}{*}{2.0} & $\mathrm{q}_{\mathrm{e}}$ & 7.75 & 8.84 & 9.3 & 10.27 & 6.12 & 6.78 & 4.5 & 1.1 \\
\hline & $\mathrm{q}_{\mathrm{s}}$ & 9.23 & 7.88 & 7.21 & 6.78 & 6.88 & 5.67 & 3.33 & 2.02 \\
\hline & $\mathrm{e}$ & 0.191 & 0.109 & 0.225 & 0.339 & 0.124 & 0.163 & 0.260 & 0.836 \\
\hline \multirow[t]{2}{*}{2.5} & $\mathrm{q}_{\mathrm{s}}$ & 8.88 & 11.12 & 7.89 & 6.98 & 4.87 & 4.45 & 2.99 & 2.10 \\
\hline & $\mathrm{e}$ & 0.128 & 0.039 & 0.153 & 0.136 & 0.186 & 0.030 & 0.488 & 0.981 \\
\hline
\end{tabular}

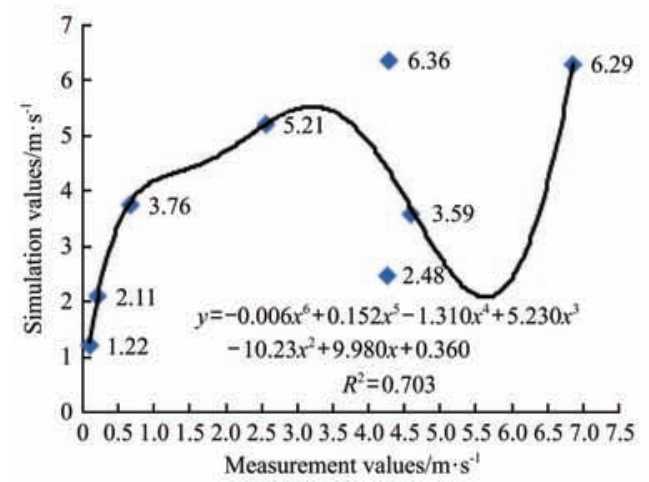

a. $h=0.5 \mathrm{~m}$

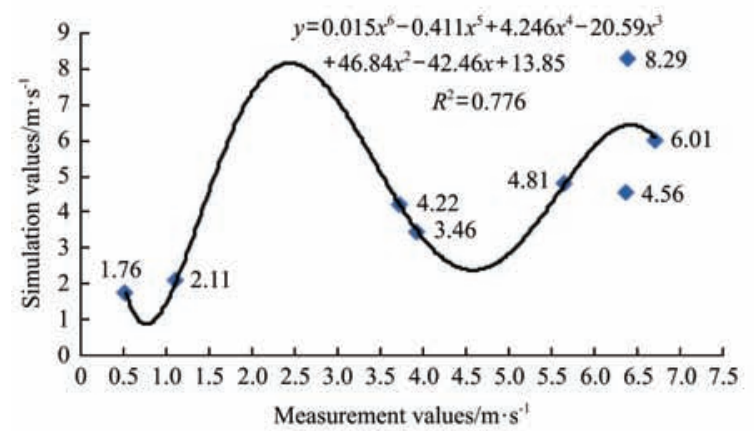

c. $h=1.5 \mathrm{~m}$

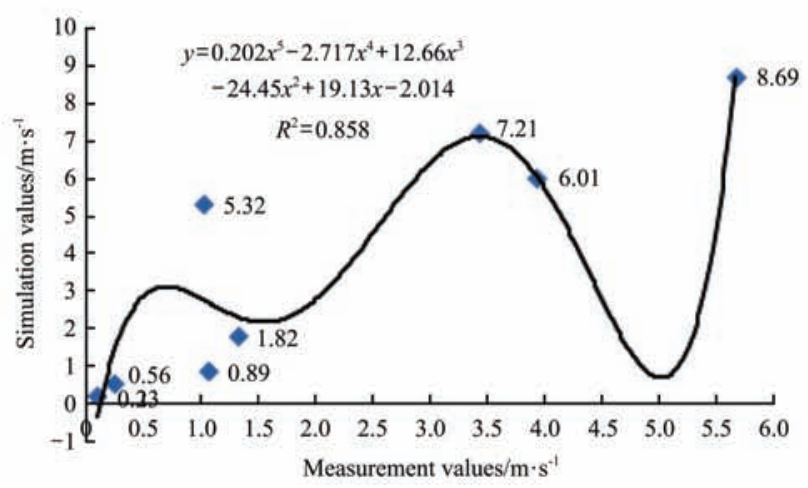

b. $h=1.0 \mathrm{~m}$

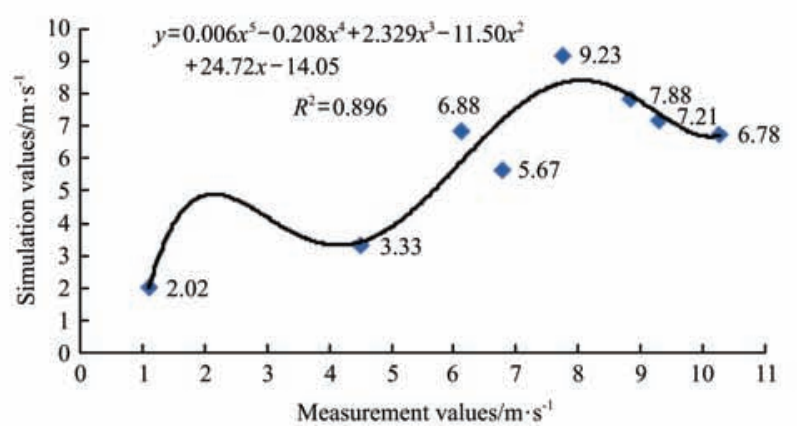

d. $h=2.0 \mathrm{~m}$

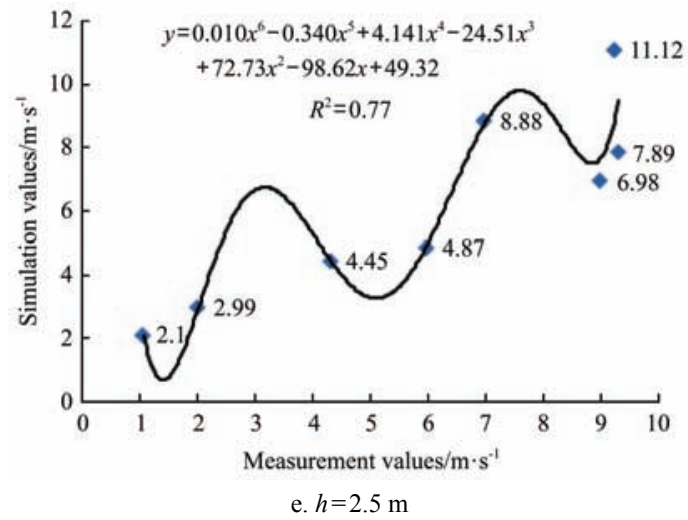

Figure 13 Polynomial fitting curves 
Sextic polynomial was chosen to fit the data except the $1.0 \mathrm{~m}$ and $2.0 \mathrm{~m}$ heights (negative values appeared with sextic polynomial). When the height was $0.5 \mathrm{~m}$, the $R^{2}$ was smallest indicating a higher degree of idealized for the simulation data, while other cases the $R^{2}$ all exceeded 0.75 , which indicated that the polynomial equations had a certain reference value for the relationship between simulation and experimental data.

\section{Conclusions and discussion}

The downwash distribution of the single-rotor UAH $\mathrm{N}-3$ was studied through the CFD simulation by improving the software model and measurement experiment verification by designing a novel device. Several conclusions can be drawn: The downwash covers approximately a circle area of $3.0 \mathrm{~m}$ radius (about 2 times of the rotor radius size) effectively with $0.5 \mathrm{~m} / \mathrm{s}$ as the boundary velocity, which provides a reference to determine the aerial spraying width. The simulation and experimental results both show that the downwash velocity changes from small to bigger then smaller as the radial distance increases, and the downwash with big velocity value relatively cover about a quarter to three quarters of the rotor radius, and as the longitudinal altitude increases the downwash velocity exists a local minimal interval and a local maximum interval. The conclusion could be referred to optimize the aerial spraying flight height to ensure that the downwash could assist the penetration and deposition of the droplets efficiently. The simulation velocity values are bigger than the experimental ones overall because of some necessary assumptions. The data obtained by simulation and experiment are compared and fit, the overall relative error margins are less than 0.7 , and the polynomial fitting coefficients of determination $R^{2}$ values are quite high, indicating the polynomial equations' reference value for the relationship between simulation and experimental data. Also in the study in order to facilitate the construction of the model, some necessary assumption are made, so that some part of the downwash distribution especially the part closed to the ground has not been explored clearly enough. In addition, during the experiments only the most concerned downwash distribution are studied by height and direction, the further exploration subsequently for the downwash distribution integrity will be needed.

\section{Acknowledgements}

The authors gratefully acknowledge the National Natural Science Foundation of China (No.31701327), the National Key Research and Development Program of China (No. 2017YFD0701000) and the Natural Science Foundation of Jiangsu Province, China (BK 20151074). This work was also part of the National Key Research and Development Plan: High Efficient Ground and Aerial Spraying Technology and Intelligent Equipment (Grant No. 2016YFD0200700).

\section{[References]}

[1] Xue X Y, Liang J, Fu X M. Prospect of aviation plant protection in China. Chinese Agricultural Mechanization, 2008; 5: 72-74. (in Chinese)

[2] Xue X Y, Qin W C, Sun Z, Zhang S C, Zhou L X, Wu P. Effect of N-3UAV spraying methods on the efficiency of insecticides against plant hoppers and Cnaphalocrocis medinalis. Acta Phytophyl Acica Sinca, 2013; 40(3): 273-278. (in Chinese)

[3] An J L, Xiang W L, Han Z W, Xiao K T, Wang Z F, Wang X $\mathrm{H}$, et al. Validation of the Institute of Atmospheric Physics emergency response model with the meteorological towers measurements and SF6 diffusion and pool fire experiments. Atmospheric Environment, 2013; 81: 60-67.

[4] Yang F B, Xue X Y, Zhang L, Sun Z. Numerical simulation and experimental verification on downwash air flow of six-rotor agricultural unmanned aerial vehicle in hover. Int J Agric \& Biol Eng, 2017; 10(4): 41 - 53.

[5] Qin W C, Xue X Y, Zhou L X, Zhang S C, Sun Z, Kong W, Wang B K. Effects of spraying parameters of unmanned aerial vehicle on droplets deposition distribution of maize canopies. Transactions of the CSAE, 2014; 30(5): 50-56. (in Chinese)

[6] Xue X, Lan Y, Sun Z, Chang C, Hoffmann W C. Develop an unmanned aerial vehicle based automatic aerial spraying system. Computers and Electronics in Agriculture, 2016; 128: 58-66.

[7] Xue X Y, Tu K, Qin W C. Drift and deposition of ultra-low altitude and low volume application in paddy field. Int $\mathrm{J}$ Agric \& Biol Eng, 2014; 7(4): 23-28.

[8] Zhang S C, Xue X Y, Qin W C, Sun Z, Ding S M, Zhou L X. Simulation and experimental verification of aerial spraying 
drift on N-3 unmanned spraying helicopter. Transactions of the CSAE, 2015; 31(3): 87-93. (in Chinese)

[9] Johnson W. Helicopter theory. Princeton University Press, 1980.

[10] Gessow A, Myers G C Jr. Aerodynmics of the helicopter. New York: Frederick Ungar Publishing Co., 1985.

[11] Wang $\mathrm{S} \mathrm{C}, \mathrm{Xu} \mathrm{Z}$. The simplified calculation methods of rotor aerodynamic load. Acta Aeronautica ET Astronautica Sinica, 1982; 3(2): 1-17. (in Chinese)

[12] Miller R H. A simplified approach to the free wake analysis of a hovering rotor, Proceedings of 7 th European Rotorcraft Forum, 1981.

[13] Wang $\mathrm{S} \mathrm{C}, \mathrm{Xu} \mathrm{G} \mathrm{H.} \mathrm{Process} \mathrm{of} \mathrm{helicopter} \mathrm{rotor}$ aerodynamics. Journal of Nanjing University Aeronautics and Astronautics, 2001; 33(3): 203-211. (in Chinese)

[14] Zhao Q J, Xu G H. Numerical simulations for the downwash flowfield of helicopter rotors. Journal of Nanjing University of Science and Technology, 2005; 29(6): 669-678. (in Chinese)

[15] Ren L F, Zhang J Z, Shan Y. Effect of helicopter rotor downwash air flow on exhaust plume flow. Journal of Aerospace Power, 2015; 29(1): 51-58. (in Chinese)

[16] Xue X Y, Lan Y B. Agricultural aviation application in USA. Transactions of the CSAM, 2013; 44(5): 194-201. (in Chinese)

[17] Lee Y. On overset grids connectivity and automated vortex tracking in rotorcraft CFD. Maryland: Department of Aerospace Engineering, University of Maryland at College Park, 2008 .
[18] Lee T E. Design and performance of a ducted coaxial rotor in hoer and forward flight. Maryland: Department of Aerospace Engineering, University of Maryland, 2011.

[19] Thomson S J, Womac A R, Mulrooney J E. Reducing pesticide drift by considering propeller rotation effects from aerial application near buffer zones. Sustainable Agriculture Research, 2013: 2(3): 41-51

[20] Li J Y, Zhou Z Y, Hu L, Zang Y, Xu S, Liu A M, et al. Optimization of operation parameters for supplementary pollination in hybrid rice breeding using round multi-axis multi-rotor electric unmanned helicopter. Transactions of the CSAE, 2014; 30(11): 1-9. (in Chinese)

[21] Wang P, Hu L, Zhou Z Y, Yang W S, Liu A M, Luo X W, et al. Wind field measurement for supplementary pollination in hybrid rice breeding using unmanned gasoline engine single-rotor helicopter. Transactions of the CSAE, 2013; 29(3): 54-61. (in Chinese)

[22] Hu L, Zhou Z Y, Luo X W, Wang P, Yan Y A, Li J Y. Development and experiment of a wireless wind speed sensor network measurement system for unmanned helicopter. Transactions of the CSAM, 2014; 45(5): 194-201. (in Chinese)

[23] Zhang B, Tang Q, Chen L P, Xu M. Numerical simulation of wake vortices of crop spraying aircraft close to the ground. Biosystems Engineering, 2016; 145: 52-64.

[24] Molari G, Benini L, Ade G. Design of a recycling tunnel sprayer using CFD simulations. Trans. ASAE, 2005; 48(2): 463-468.

[25] Tsay J, Ozkan H E, Brazee R D, Fox R D. CFD simulation of moving spray shields. Trans. ASAE, 2002; 45(1): 21-26. 